
\title{
$\begin{array}{ll}\text { Research Square } & \begin{array}{l}\text { Preprints are preliminary reports that have not undergone peer review. } \\ \text { They should not be considered conclusive, used to inform clinical practice, } \\ \text { or referenced by the media as validated information. }\end{array}\end{array}$
}

\section{The Relationship Between Ultrasound and Semen Parameters in Male Infertility: Size and Elasticity Matters!}

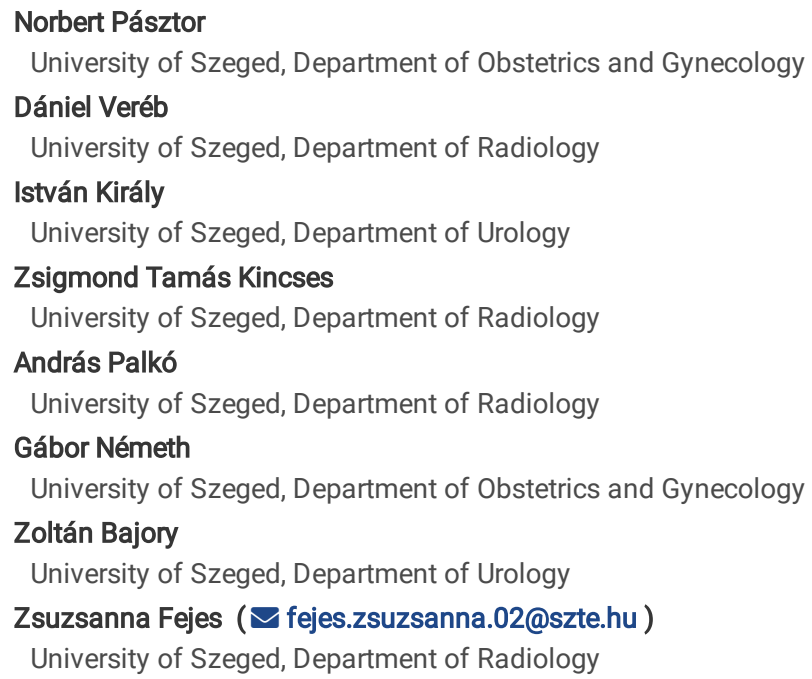




\section{Abstract}

\section{Objectives}

Testicular ultrasound is important in the assessment of male infertility and adding strain elastography to the protocol can help clarify the underlying etiology.

\section{Material and Methods}

78 participants (64 with abnormal, 14 with normal semen parameters) underwent sperm analysis and testicular US including strain elastography. Semen parameters, testicular volumes, strain elastography parameters were compared between groups using independent samples T-tests, least squares regression, Spearman's rank correlation.

\section{Results}

Participants with abnormal semen parameters exhibited reduced testicular volumes (left: $p<0.0003$, right: $p<0.0004$ ) and epididymis diameters (left: $p<0.01$, right: $p<0.006$ ), and higher strain ratios (left: $p<0.006$, right: $p<0.001$ ). The first PLS latent variable explained a significant amount of variance in semen parameters $(p<0.0002)$ and showed that bilateral strain ratios, left testicular volume and left appendix size were the most important predictors. Elevated strain ratios came with reduced sperm cell number (right: $R=-0.45, p<0.0001$; left: $R=-0.46, p<0.0001$ ), sperm concentration (right: $R=-0.46, p<0.0001$; left: $R=-0.48$, $p<0.0001$ ), total (right: $R=-0.39, p<0.001$ ) and progressive motility (right: $R=-0.4, p<0.001$ ), non-motility (left: $R=-0.37, p<0.003)$ normal morphology (right: $R=-0.37, p<0.003)$.

\section{Conclusion}

We confirm previous reports of correlation between sperm parameters, testis volume and epididymis diameter. Furthermore, we show that strain elastography parameters are strong predictors of semen parameters.

\section{Introduction}

About $20 \%$ of couples in Hungary do not achieve pregnancy within one year and seek medical treatment for infertility. One in eight couples encounters problems when attempting to conceive the first child and one in six when attempting to conceive a subsequent child. In Hungary, the proportion of involuntarily childless couples is estimated at $24 \%$, one in four couples has infertility problems [2]. Three percent of women who are currently trying to conceive remain involuntarily childless, while $6 \%$ of women with previous delivery are not able to have as many children as they would wish. In $50 \%$ of involuntarily childless couples, a male-infertility-associated factor is found, usually together with abnormal semen parameters. For this reason, all male patients belonging to infertile couples should undergo medical evaluation by a trained specialist in male reproduction [1].

A focused evaluation of the male patient must always be undertaken and should include: a medical and reproductive history; physical examination; semen analysis - with strict adherence to World Health Organization (WHO) reference values for human semen characteristics; and hormonal evaluation. Other investigations (e.g. ultrasound) may be required depending on the clinical features and semen parameters [1].

Ultrasound is the first-line modality for imaging the scrotum, the extra- and intratesticular lesions. It is a relatively fast, harmless, cost-effective method. The testicular abnormalities characterized, observed by an ultrasound examination (e.g., intratesticular mass of low echogenicity compared to normal testicular tissue or increased vascularization suggestive of inflammation) are closely related to the patient's symptoms and clinical picture. [3]. A high-frequency linear array transducer is used, to achieve high spatial resolution of the scrotal contents. Color-Doppler or power-Doppler measurements are also performed to detect, evaluate and differentiate intratesticular lesions. According to the male infertility guidelines scrotal ultrasound may be helpful to distinguish obstructive and non-obstructive azoospermia and may demonstrate inhomogeneous testicular echotexture and microlithiasis [1].

Recently, US strain elastography (SE) has become available as an additional function in many ultrasound equipments. SE is a non-invasive, real-time method, and provides information about certain tissue characteristics. SE was first described in the 1990s [4]. It measures tissue elasticity: the resistance of the tissue to deformation force, and its return to the original status. Elasticity and the deformation models can be described by Hooke's law and the Young modulus can be calculated. The estimation of the strain ratio is a semi-quantitative measurement. In a region of interest (ROI) it shows the ratio of strain measured in a normal area of reference to strain in a target lesion [5]. During the measurement strain elastographic response of the testicular tissue measuring the elasticity (elastogram) is superimposed on the B-mode image.

The relationship between testicular elasticity, serum FSH level and varicocele was described earlier [6]. The strain ratio of the testis, especially the left side, is elevated in case of varicocele, well before the atrophy of the testis. Consequently, SE could be useful to detect the damage caused by varicocele [7]. The testicular stiffness and semen parameters are known to be connected too. A negative correlation was found between the semen volume, sperm morphology and motility, and testicular stiffness [8].

Several studies found connection between the US parameters (volume, echogenity) and the qualitative and quantitative features of semen. However, US parameters alone are not able to identify testicular pathology and the correlation is not unequivocal. The traditional linear regression analysis could not predict unambiguously the importance of the variables. The non-parametric partial least square (PLS) approach, handling the problem of collinearity as well, is able to distinguish a pattern of parameters best predicting the variable in question. Therefore, we intended to identify the most predictive ultrasound parameters for the assessment of male infertility in this multiparametric study. 
Various ultrasound parameters of B-mode US and strain-elastography were related to sperm features (motility and morphology) and semen quality (total sperm count sperm concentration, total motility or sperm morphology). The aim is to study to choose one of US parameters, which reflect the beast the abnormal semen.

\section{Materials And Methods}

For this study, infertile men were recruited between December 2017 and June 2019 at the Andrology Unit of the Department of Urology and Department of Obstetrics and Gynecology, University of Szeged. For the control group, healthy volunteers from university staff and relatives were recruited. Abnormal semen and pathological gray-scale ultrasound (for details see below) of the testis were exclusion criteria from the control group.

Study population

Patients were excluded from the statistical analysis in case of present or anamnestic testicular tumor, intratesticular cyst, present acute orchitis/epididymitis, and hydrocele. Only normally descended testicles were measured.

All procedures followed were in accordance with the ethical standards of the Helsinki Declaration of 1975, as revised in 2000 . The study protocol was approved by the Regional and Institutional Human Medical Biological Research Ethics Committee, University of Szeged (No.: 56/2018-SZTE). Informed consent was gained from all participants.

Sperm analysis

For all the patients, a physical examination and a sperm analysis were performed. Semen was analyzed according to the Fifth Edition of World Health Organization (WHO) Manual for the examination of human semen. Sperm concentration, total sperm count, total sperm motility, and progressive and nonprogressive motility were counted with phase-contrast optics at $\times 200$ magnification in a Makler counting chamber (FertiCAD Kft., Budapest, Hungary), as described in the WHO laboratory manual for the examination of human semen. Sperm morphology was determined at $\times 1,000$ magnification with oil immersion after Diff-Quik staining (Diff-Quik Staining Set, Medion Diagnostics AG, Düdingen, Switzerland). Head, neck, mid-piece, tail and multiple morphological abnormalities were noted separately. In case of abnormal semen parameters, a control sample was collected and analyzed 3-4 weeks later. If the result of the control sperm analysis was abnormal again, hormonal evaluation and ultrasound examination of the testis were performed. In certain cases, ultrasound examination of the prostate and vesicular seminales were added. When sperm concentration was less than $10 \times 10^{6} / \mathrm{Ml}$ karyotyping and below $5 \times$ $10^{6} / \mathrm{Ml}$ screening for azoospermia factor microdeletion of the Y chromosome (AZFa /Sy84,86/; AZFb /Sy127,134/; AZFc /Sy254,255/ regions) were also performe.

Ultrasonography, imaging and measuring technique

The ultrasound examinations were performed on a GE Logiq E9 machine (General Electric Healthcare) with a $15 \mathrm{MHz}$ linear probe. At first, grey-scale, and color and Power Doppler ultrasonographic imaging was used to analyze testicular morphological characteristics and detect intratesticular abnormalities. Testicular volumes were calculated by multiplying the three longest diameters $(\mathrm{L} \times \mathrm{T} \times \mathrm{AP})$ using the correction factor of 0.71 , since this formula provides results similar to those obtained by orchidometry. The largest diameter of the head of epididymis was measured on both sides, the presence and length of appendix testis were noted, the presence and grade of varicocele according to Sarteschi were described.

Next we performed strain elastosonographic imaging to measure the testicular tissue elasticity. For the elastosonography, the same ultrasound equipment and linear transducer were used. The testicles were positioned on a rigid paper sheet to optimize the strain elastosonographic imaging. The probe was adjusted at the perpendicular position to the testis, displaying the largest possible cross-section, and avoiding the mediastinum testis, because of its different elasticity. Freehand technique was used. When the radiologist applies slight pressure with the transducer, a compression bar appears on the screen, indicating the appropriate pressure by a green color. When this slight pressure is stable for at least 5-10 s, the radiologist should use this frame for subsequent analysis. In normal cases, a homogenous testis with normal size and in absence of any intratesticular lesions is detected by grey-scale imaging. The testicular structure is also visualized by the elastogram, which is a color-coded picture of the tissue elasticity (Fig. 1.). Tissue stiffness is encoded from red (soft) to blue (rigid) according to the standard settings of the US equipment. The color-coded image of the normal testis is homogeneous, surrounded by a relatively harder ring, as representing the tunica albuginea of the testis with less relative strain (Fig. 2.). During the semi-quantitative analysis the region of interest (ROI) was positioned on the midline of the testis. Strain ratio was calculated as the ratio of the stiffness of the peritesticular fat and testicular parenchyma [8].

\section{Statistical analysis}

For the statistical analysis of group differences, two-tailed independent samples T-tests were used and Bonferroni-correction was performed to correct for multiple comparisons. Mann-Whitney U-test was used to detect differences between the strain ratios of the left and right testis.

For a subset of the patients $(n=38)$, we calculated Spearman-Rank correlation between levels of TSH, prolactin, testosterone, SHBG, FSH and LH, and ultrasonography parameters, and repeated the same for functional measures.

For the partial least squares regression, we chose 10 measures as explanatory or descriptor variables: participant age, volumes and strain ratios of the bilateral testes and epididymis, and the presence and size of the appendix testis on both sides. Since some of the variables exhibit a non-normal probability distribution and, especially in case of the bilateral parameters, collinearity, we opted for a partial least squares regression (PLS) approach. The PLS method is based on a linear decomposition of the predictor and response matrix, optimized so that the covariance between the predictor and response component matrix is maximal and the residual terms are minimal[9]. We designated sperm cell number, sperm cell concentration, functional measures of sperm cells (measures

Page $3 / 9$ 
of motility, ratio of cells with abnormal morphology and specific measures of morphology, such as alterations of the head, neck, midpiece and tail) and the first principal component of these measures (referred to as composite score later on) as dependent variables. We tested latent variables in the PLS for significance using a permutation-based approach: the dependent variable matrix was shuffled 5000 times and for each permutation, the covariance matrix of the predictor and response factors were computed, thus we were able to obtain a null distribution for the eigenvalue corresponding to the tested latent variable. We calculated exact p-values according to Phipson and Smyth[10]. To correct for multiple hypothesis testing, obtained p-values were corrected according to the Benjamini-Hochberg method of false discovery rate control[11]. We also calculated Variable Importance for Projection (VIP) scores for the explanatory variables, which quantifies the extent to which a given variable takes part in explaining the response variable[12]. The statistical analyses were conducted using the Statistics and Machine Learning Toolbox included in MATLAB (version R2014B, MathWorks, Inc.).

\section{Results}

Altogether 64 males were recruited in the study group and 14 men in the control group. The average age was 37.1 years (range: 22-54) of the study group, and 36.3 years $(25-42)$ for the controls $(p=0.63)$. In the study group, $21,9 \%$ cases were presented with azoospermia by the semen analysis, $15.6 \%$ with cryptozoospermia, $31.2 \%$ of infertile men had oligozoospermia was found in $6.3 \%$, asthenozoospermia in $12.5 \%$, and oligo-asthenozoospermia was the diagnosis in $12.5 \%$ of cases. In the case of azoospermia micro-TESE surgery was performed, and pathological Johnsen score is correlated with the strain ratio in patient suffered from non-obstructive azoospermia. The prevalence of varicocele was $29.6 \%$ in the study and $14.3 \%$ in the control group, which was not significant $(p=0.33)$, though presented a 2.53 -fold odds ratio. No statistical difference was observed between patients with and without varicocele regarding age $(p=0.16)$; testicular volumes $(p=0.77$ for the right, $p=0.25$ for the left testis); sperm concentration $(p=0.74)$; total sperm number $(p=0.88)$; total motility $(p=0.78)$, progressive motility $(p=0.66)$ and normal morphology $(p=0.93)$. No significant difference was detected between the strain ratios of the two testis in patients with varicocele $(p=0.39)$, without varicocele $(p=0.41)$ and in the whole study population $(p=0.28)$. The testicular volumes, the diameters of epididymis head on both sides and the sperm parameters were statistically different between the study and the control group. The main data of the participants are summarized in Table 1.

Table 1

Main attributes of the study and control group.

\begin{tabular}{|c|c|c|c|c|}
\hline & Study group $(n=64)$ & Control group $(n=14)$ & p value & Corrected p-value threshold \\
\hline Age (years) (mean, range) & $37.1(22-54)$ & $36.3(25-42)$ & 0.63 & - \\
\hline Sperm concentration (million/ml) (mean +/-SD, median) & $1.5+/-3.5(0)$ & $57.5+/-29.4(47)$ & $<0.0001^{\square}$ & 0.007 \\
\hline Total sperm number (million/ejaculate) (mean +/- SD, median) & $6.3+/-15.5(0)$ & $242.2+/-160.9(220)$ & $<0.0001^{\square}$ & 0.007 \\
\hline Total sperm motility (\%) (mean +/- SD, median) & $28.6+/-27.4(24.5)$ & $73.6+/-8.5(70.5)$ & $<0.0001^{\square}$ & 0.007 \\
\hline Progressive motility (\%) (mean +/- SD, median) & $14.3+/-15.7(10)$ & $53.4+/-16.4(50)$ & $<0.0001^{\square}$ & 0.007 \\
\hline Non-progessive motility (\%) (mean +/- SD, median) & $14.3+/-14.0(14)$ & $21.6+/-12.7(19.5)$ & 0.08 & 0.007 \\
\hline Normal morphology (\%) (mean +/- SD, median) & $2.0+/-2.4(1.5)$ & $19.7+/-8.2(21)$ & $<0.0001^{\square}$ & 0.007 \\
\hline Presence of microlithiasis (\%) & 17.2 & 0 & 0.2 & - \\
\hline Presence of varicocele grade II-III (\%) & 29.6 & 14.28 & 0.33 & - \\
\hline Presence of appendix testis (\%) & 14 & 0 & 0.2 & - \\
\hline Right testis volume $(\mathrm{ml})($ mean $+/-\mathrm{SD})$ & $11.9+/-6.0$ & $19.1+/-4.2$ & $<0.0001^{\square}$ & 0.01 \\
\hline Left testis volume $(\mathrm{ml})($ mean $+/-\mathrm{SD})$ & $11.1+/-5.3$ & $17.7+/-4.6$ & $<0.0001^{\square}$ & 0.01 \\
\hline Right epididymis head diameter $(\mathrm{mm})($ mean +/- SD) & $9.7+/-2.3$ & $12.1+/-2.6$ & $0.001^{\square}$ & 0.01 \\
\hline Left epididymis head diameter $(\mathrm{mm})($ mean $+/-\mathrm{SD})$ & $8.9+/-2.4$ & $11.4+/-3.5$ & $0.002^{\square}$ & 0.01 \\
\hline
\end{tabular}

In the study group, higher strain ratios were found for both testis $(1.687+/-0.539$ vs. $1.114+/-0.156, p<0.001$ for right testis; $1.800+/-0.657$ vs. $1.193+/-$ $0.220, p<0.006$ for left testis) compared to the control patients, after correction for multiple comparisons.

Significant correlations were found among the sperm parameters, as presented in Fig. 3. To eliminate the effect of these cross-correlations, we used PLS regression as a multivariate method for further calculations. According to the VIP scores, the strain ratios of both testes, the volume of left testis and the size if the left appendix were identified as the most important factors associated with the results of semen analysis. In this study we focused on the testicular strain ratios. The optimal model order for the PLS regression included 2 latent variables, but the second latent variable was not deemed significant $(p<0.102)$ as per the permutation test for any response variable, and thus we excluded it from further analysis. The first latent variable explained a significant amount of variance in sperm cell number and concentration, and also progressive motility and morphology parameters $(p<0.0002)$. The VIP scores and the percentage of variance by the two latent variables are showed in Fig. 4 . The correlations between the sperm parameters and the strain ratios of the testis are showed in Table 2, and the correlation coefficients between testicular strain ratios and sperm parameters are presented in Fig. 4. 
After correcting for multiple hypothesis testing in the same way as described in the PLS section, the volumes of bilateral testes correlated negatively with FSH levels $(R=-0.56, p<0.007$ and $R=-0.67, p<0.0003)$. As for the functional measures, sperm cell concentration and sperm cell count decreased with increasing FSH $(R=-0.52, p<0.029$ and $R=-0.58, p<0.01)$ (not shown in the tables).

Table 2: Correlations between the testicular strain ratios and sperm parameters in pathozoospermic men $(n=64)$ Strain ratio was calculated as the ratio of the stiffness of the peritesticular fat and testicular parenchyma [8]. Semen was analyzed Partial least squares regression was used to describe the covariance between strain ratios of the testis and sperm parameters. Spearman's rho coefficient was calculated. Corrections for multiple comparisons were carried out according to Bonferroni.

\begin{tabular}{|c|c|c|c|c|c|c|c|c|c|c|c|}
\hline & & $\begin{array}{l}\text { Sperm cell } \\
\text { concentration }\end{array}$ & $\begin{array}{l}\text { Sperm } \\
\text { cell } \\
\text { number }\end{array}$ & $\begin{array}{l}\text { Total } \\
\text { motility }\end{array}$ & $\begin{array}{l}\text { Progressive } \\
\text { motility }\end{array}$ & $\begin{array}{l}\text { Non- } \\
\text { progressive } \\
\text { motility }\end{array}$ & $\begin{array}{l}\text { Non- } \\
\text { motile } \\
\text { sperm } \\
\text { cell } \\
\text { number }\end{array}$ & $\begin{array}{l}\text { Percent of } \\
\text { normal } \\
\text { morphology }\end{array}$ & $\begin{array}{l}\text { Percent of } \\
\text { abnormal } \\
\text { morphology }\end{array}$ & $\begin{array}{l}\text { Percent of } \\
\text { head } \\
\text { abnormality }\end{array}$ & $\begin{array}{l}\text { Perc } \\
\text { neck } \\
\text { midr } \\
\text { abnc }\end{array}$ \\
\hline \multirow{3}{*}{$\begin{array}{l}\text { Right } \\
\text { testis } \\
\text { strain } \\
\text { ratio }\end{array}$} & $\begin{array}{l}\text { Spearman's } \\
\text { rho }\end{array}$ & -0.451 & -0.462 & -0.395 & -0.404 & -0.340 & -0.259 & -0.370 & -0.131 & -0.157 & $-0.2 \mathrm{c}$ \\
\hline & p-value & $0.0001^{\square}$ & $0.0001^{\square}$ & $0.001^{\square}$ & $0.0009^{\square}$ & 0.0058 & 0.038 & $0.0025^{\square}$ & 0.2991 & 0.2151 & 0.01 \\
\hline & $\begin{array}{l}\text { corrected p- } \\
\text { value } \\
\text { threshold }\end{array}$ & 0.0038 & & & & & & & & & \\
\hline \multirow{3}{*}{$\begin{array}{l}\text { Left } \\
\text { testis } \\
\text { strain } \\
\text { ratio }\end{array}$} & $\begin{array}{l}\text { Spearman's } \\
\text { rho }\end{array}$ & -0.456 & -0.479 & -0.296 & -0.315 & -0.238 & -0.371 & -0.285 & -0.230 & -0.132 & $-0.2 i$ \\
\hline & p-value & $0.0001^{\square}$ & $<0.0001^{\square}$ & 0.0174 & 0.0111 & 0.0576 & $0.0025^{\square}$ & 0.0222 & 0.0665 & 0.2979 & 0.02 \\
\hline & $\begin{array}{l}\text { corrected p- } \\
\text { value } \\
\text { threshold }\end{array}$ & 0.0038 & & & & & & & & & \\
\hline
\end{tabular}

a: statistically significant correlation, when p-value is equal or lower than the corrected p-value threshold.

\section{Discussion}

Testicular ultrasound examination is very important in the investigation of male infertility because of its sensitivity and cost-effectiveness. Ultrasound examination has a widespread use in case of differential diagnosis of the intratesticular lesion especially if non-palpable. The multparametric ultrasound could help with the differentiation malignant and benignant intratesticular lesions too[13]. The accessibility and non-invasibilty makes the US an excellent tool in evaluating the male infertility, however it is crucial to know which of the US parameter are the best predictors for semen analysis. To our knowledge, this is the first study which attempted to identify the most characteristic ultrasound parameters of male infertility with an exploratory multivariate approach.

Age is known to be associated with the changes of testicular parenchyma that are know to contribute to the elasticity of the testis too: increasing tubular sclerosis, Leydig-cell hyperplasia and focal monoclonal inflammation. Tubular ectasia of the rete testis and capsular smooth muscle hyperplasia may also occur with increasing age [15]. The peritubular fibrosis restricts the seminiferous tubules and may cause declension of spermatogenesis [16]. These pathological changes may lead to be "more rigid" than normal testis, which could be detected with strain elastography [17].

Our multivariate, exploratory analysis revealed that elasticity of the testes and the volume of the left testis and the appendix are the most significant US predictors of the pathological semen and sperm cell features. We found correlation between the serum FSH levels and size of the testes and in turn significant correlation between the semen and sperm cell features and FSH levels.

Our results are consistent with other studies. The smaller testicular volume is associated with decreased sperm motility and count, and the high FSH and LH levels affecting negatively the male fertility, and have been related to smaller testicular volume. [1, 14]

In line with previous studies we tried to find the role of strain elastrography in US examination during the investigation of male infertility [8][18]. The results demonstrate two thing, at first left testis is more important than the right in terms of fertility. Second one US parameter is not enough, but strain elastography by experienced radiologist may shorten the time of the examination and clarify the expected result.

We have shown that the size of the left testis and appendix are determining, characterizing the male fertility more than the right. There is a natural difference between left and right testicular volume in the healthy population. This is because in humans, the testis on the right seems to develop faster than on the left. In females, this phenomenon is the opposite, with the ovaries developing first on the left [19]. A study shows a positive Testicular Asymmetry Index (TAl) for the entire population and for all Tanner stages, excluding TSG1, meaning there is a natural difference between left and right testicular volume in a healthy adolescent population, left testis being smaller. Small testicular volumes and high testicular volume differences are associated with poor semen analysis outcomes. Large testicular volume differences are expected to be seen in patients with unilateral inguinoscrotal disorders. [20]

Ultrasound examination, in addition to knowledge of urological anamnestic data and hormone levels, is useful for diagnosing semen abnormalities such as determining the type of male infertility. Strain elastography may provide additional information on scrotal and testicular conditions [21][22]. Strain elastography-supplemented ultrasonography is better correlated with the semen parameters than the B-mode US examination. The strain elastographysupplemented US examination could help the urologist to choose the most accurate treatment for the patients. Male infertility is a complex, multi-factorial 
disease, but an appropriate ultrasound examination can help to establish and confirm the diagnosis. The diagnosis and differentiation between obstructive and non-obstructive azoospermia could be confirmed by strain elastography, the elastogram showing lower testicular rigidity, which could predict the semen parameters and in the case of micro-TESE (microsurgical testicular sperm extraction) operation the success of fertilization.

The main limitation of our study is the low number of participants and the few azoospermic male (both obstructive and non-obstructive azoospermic patients group) in our sample. The distinction between obstructive and non-obstructive azoospermia is important, in which medical history (cryptorchidism), levels of hormones (LH, FSH and testosterone) may help. Sonographic features that may be present with obstructive azoospermia include normal testicular size and echogenicity (testicular echogenicity is same as the thyroid gland in euthyreosis), rete testis ectasia or spermatocele. The smaller testicular volume and low echogenicity, presence of varicocele are characteristic of the non-obstructive azoospermia. In future work, investigating the connection between strain elastography and histopathological patterns of testicular biopsy in males suffering from non-obstructive azoospermia might provide further important insights into male infertility.

\section{Conclusion}

Even though the main stream of radiology concentrates on cross sectional imaging, ultrasound equipments also develops rapidly, and in case of scrotal imaging the availability, spatial resolution and the non-ionising radiation based imaging offers a unique opportunity to investigate male infertility. Our results showed that strain elastography is among the most sensitive US approaches in evaluating male infertility. The radiologist should focus on the left testicular elastogram, echogenicity and the presence of the appendix testis because of they have the most predictive value. It is sensitive for the tissue microstructure, hence it can help the andrologists and urologists to characterize the type of male infertility.

\section{Abbreviations}

LH - Luteinizing hormone

FSH - Follicle-stimulating hormone

micro-TESE - microsurgical testicular sperm extraction

ROI - Region of Interest

PLS - Partial least squares

SE - strain elastography

SHBG - Sex hormone-binding globulin

TAl - Testicular Asymmetry Index

US - Ultrasonography

VIP - Variable Importance for Projection

WHO - World Health Organization

\section{References}

1. European Association, U. European Association of Urology Guidelines. 2020 Edition. Available via https://uroweb.org/guideline/sexual-and-reproductivehealth/ Accessed 05 April 2021.

2. Szatmári, A. et al. Men's knowledge of male infertility and the possibilities for supporting therapy: risk factors and individual responses. Journal of Reproductive and Infant Psyhology. https://doi.org/org/10.1080/02646838.2020.1724918 (2020).

3. Moreno, C. C. et al. Testicular tumors: what radiologists need to know-differential diagnosis, staging, and management. Radiographics. 35 (2), $400-415$ (2015).

4. Gennisson, J. L., Deffieux, T., Fink, M. \& Tanter, M. Ultrasound elastography: principles and techniques. Diagn Interv Imaging. 94 (5), $487-495$ (2013).

5. Sigrist, R. M. S., Liau, J., Kaffas, A. E., Chammas, C. M. \& Willmann, J. Ultrasound Elastography: Review of Techniques and Clinical Applications. Theranostics. 7 (5), 1303-1329 (2017).

6. Dede, O. et al. Elastography to assess the effect of varicoceles on testes: a prospective controlled study. Andrologia. 48 (3), $257-261$ (2016).

7. Bitkin, A. et al. Effects of varicocele on testicles: Value of strain elastography: A prospective controlled study. Andrologia. 51 (1), 13161 (2019).

8. Kucukdurmaz, F. et al. Evaluation of the diagnostic efficacy of strain elastography in infertile population with normal and abnormal semen parameters. Turk J Urol. 43 (3), 261-267 (2017).

9. AbdiH, Williams, L. J. Partial least squares methods: partial least squares correlation and partial least square regression. Methods in molecular biology. 930, 549-579 (2013).

10. Phipson, B. \& Smyth, G. K. Permutation P-values should never be zero: calculating exact P-values when permutations are randomly drawn. Stat App/ Genet Mol Biol. 9 (10), 1544-6115 (2010). 
11. Benjamini, Y. \& Hochberg, Y. Controlling the False Discovery Rate: A Practical and Powerful Approach to Multiple Testing. Journal of the Royal Statistical Society. Series B (Methodological). 57 (1), 289-300 (1995).

12. Kubinyi, H. (1993) 3D QSAR in drug design: volume 1: theory methods and applications. Vol. 1. 1993: Springer Science \& Business Media.

13. Auer, T. et al. ) Value of multiparametric US in the assessment of intratesticular lesions. 285 (2), 640-649 (2017).

14. Sakamoto, H. et al. Relationship between testicular size by ultrasonography and testicular function: Measurement of testicular length, width, and depth in patients with infertility. International Journal of Urology. 15 (6), 529-533 (2008).

15. Honore, L. H. Ageing changes in the human testis: a light-microscopic study. Gerontology. 24 (1), 58-65 (1978).

16. Apa, D. D., Cayan, S., Polat, A. \& Akabay, E. Mast cells and fibrosis on testicular biopsies in male infertility. Arch Androl. 48 (5), $337-344$ (2002).

17. Roy, C., Marini de, P., Labani, A., Leyendecker, P. \& Ohana, M. (2020) Shear-wave elastography of the testicle: potential role of the stiffness value in various common testicular diseases. Clinical Radiology 75(7): p. 560.e9-560.e17.

18. Rocher, L. et al. Testicular Shear Wave Elastography in Normal and Infertile Men: A Prospective Study on 601 Patients. Ultrasound Med Biol. 43 (4), 782789 (2017).

19. Bogaert, A. F. Genital asymmetry in men. Hum Reprod. 12 (1), 68-72 (1997).

20. Vaganee, D. et al. Beats de K, (2018) Testicular asymmetry in healthy adolescent boys.BJU Int, 122(4): p.654-666.

21. Fang, C., Huang, D. Y. \& Sidhu, P. S. Elastography of focal testicular lesions: current concepts and utility. Ultrasonography (Seoul, Korea). 38 (4), $302-310$ (2019).

22. Konstantatou, E. et al. (2019) Evaluation of intratesticular lesions with strain elastography using strain ratio and color map visual grading: differentiation of neoplastic and nonneoplastic lesions. 38(1): p. 223-232.

\section{Figures}

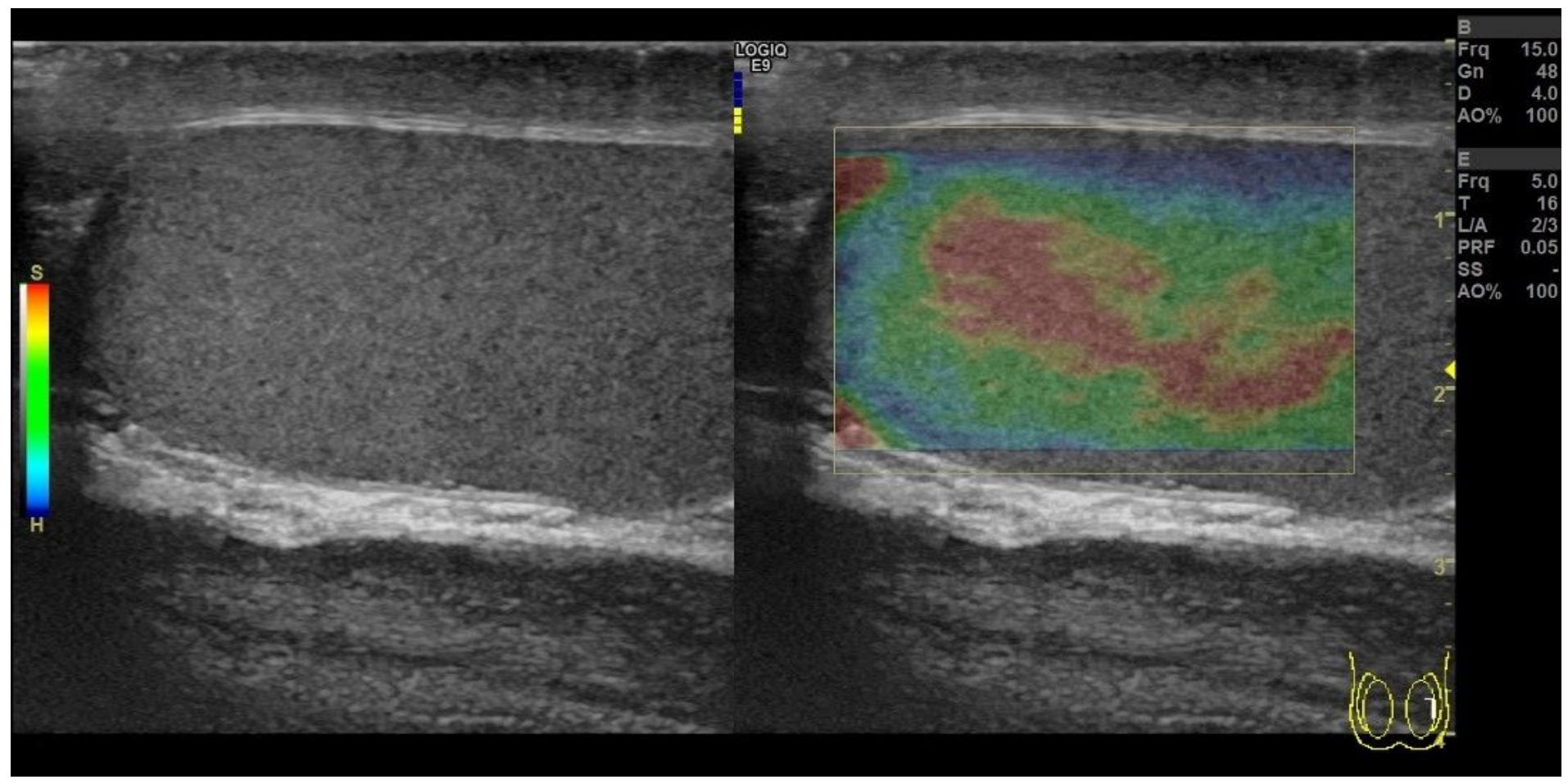

Figure 1

Strain elastography (color coded picture - elastogram) and B-mode images of the normal testis. The central parenchyma is green color with blue edges ('boundary' effect), which is surrounded by red bands. 


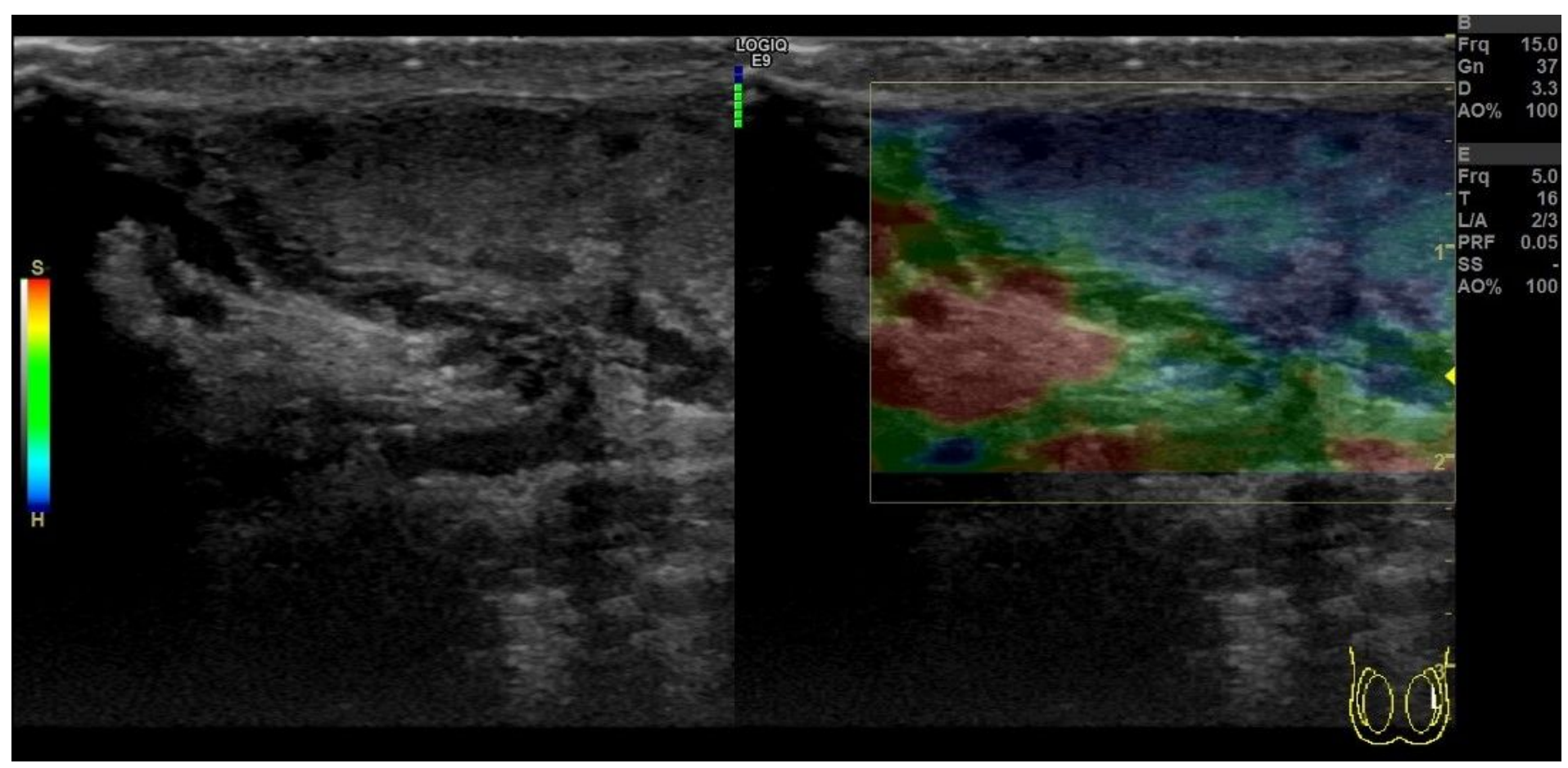

\section{Figure 2}

elastogram of testis of patient (29-year old) suffer from non-obstructive azoospermia. The central parenchyma has inhomogeneous, decreased echogenicity in the B-mode image, and blue (rigid) in the elastogram lack of 'boundary' effect.
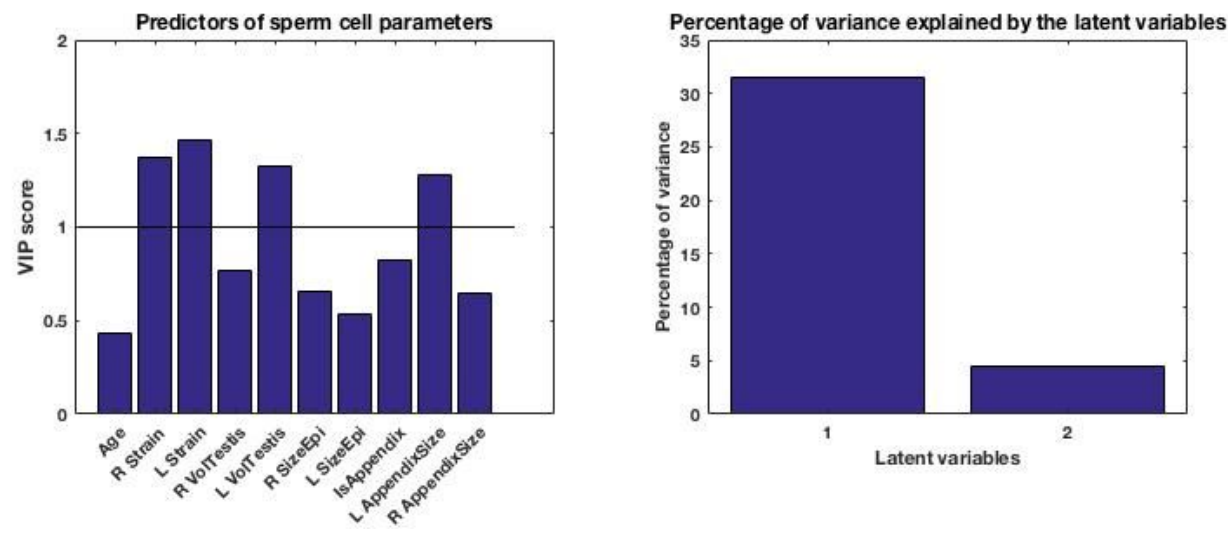

Figure 3

Cross-correlation between sperm cell parameters. The figure depicts the cross-correlation matrix of sperm cell parameters. Circle size and color shows the strength and direction of the correlation. The colorbar depicts Spearman's rank correlation coefficients. 


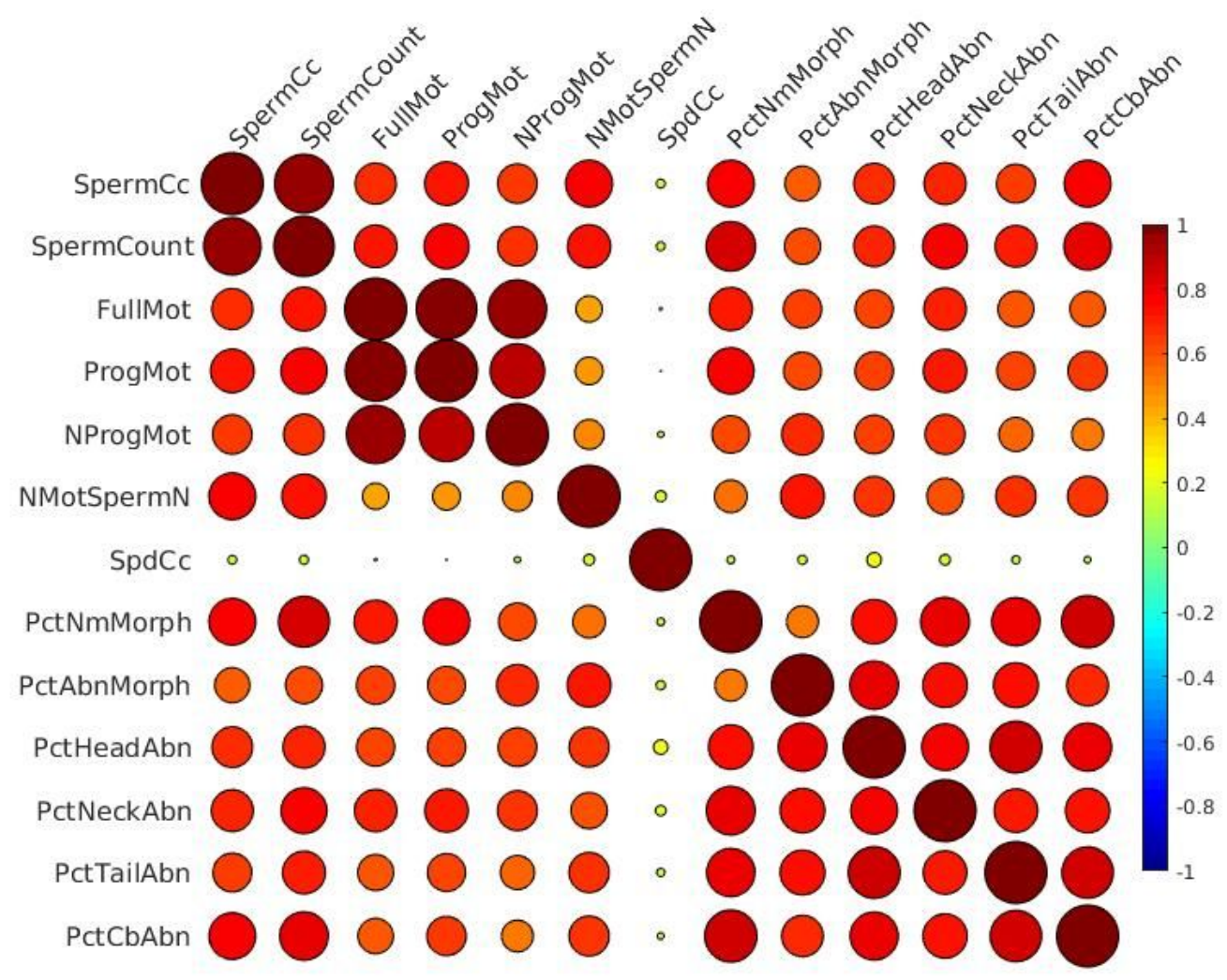

Figure 4

Cross-correlation between sperm cell parameters. The figure depicts the cross-correlation matrix of sperm cell parameters. Circle size and color shows the strength and direction of the correlation. The colorbar depicts Spearman's rank correlation coefficients. 